\title{
A Subgrid-Scale Model for the Scalar Dissipation Rate in Nonpremixed Combustion
}

\author{
A. W. Cook \\ W. K. Bushe \\ This paper was prepared for submittal to the \\ 51st Annual Meeting of the American Physical Society \\ Division of Fluid Dynamics \\ Philadelphia, PA \\ November 22-24, 1998
}

August 31, 1998

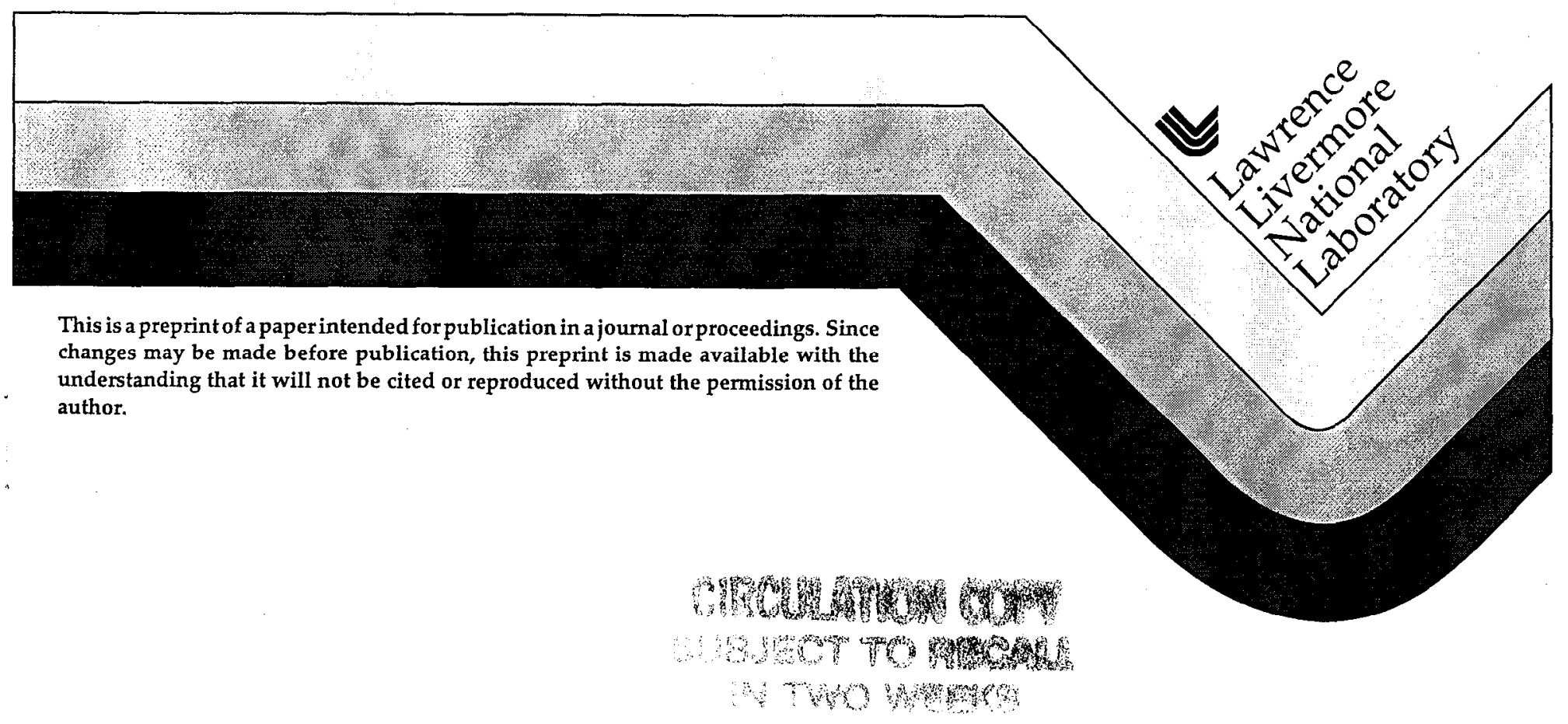




\section{DISCLAIMER}

This document was prepared as an account of work sponsored by an agency of the United States Government. Neither the United States Government nor the University of California nor any of their employees, makes any warranty, express or implied, or assumes any legal liability or responsibility for the accuracy, completeness, or usefulness of any information, apparatus, product, or process

disclosed, or represents that its use would not infringe privately owned rights. Reference herein to any specific commercial product, process, or service by trade name, trademark, manufacturer, or otherwise, does not necessarily constitute or imply its endorsement, recommendation, or favoring by the United States Government or the University of California. The views and opinions of authors expressed herein do not necessarily state or reflect those of the United States Government or the University of California, and shall not be used for advertising or product endorsement purposes. 


\title{
A subgrid-scale model for the scalar dissipation rate in nonpremixed combustion
}

\author{
By A. W. Cook ${ }^{1}$ AND W. K. Bushe
}

A subgrid-scale model is presented for the scalar dissipation rate in nonpremixed turbulent reacting flows. Inputs to the model are the filtered density, the Favrefiltered temperature and the Favre-filtered mixture-fraction. The model contains a coefficient which is determined by assuming a form for the scalar energy spectrum. Inputs to the presumed spectrum are the integral and dissipation length scales of the scalar field. These quantities are estimated locally from the Favre-filtered velocity field, resulting in a model coefficient which is spatially and temporally dependent. The model is tested a priori using data from a Direct Numerical Simulation (DNS) of a temporal reacting mixing layer. Estimated values of the dissipation rate are found in good agreement with dissipation rates computed directly from the DNS data. Furthermore, the presumed spectrum methodology is found to accurately predict the mean value of the model coefficient as well as its spatial and temporal variations.

\section{Introduction}

The Large Eddy Simulation (LES) of chemically reacting turbulent flows has become a topic of much interest in recent years. The application of LES to nonpremixed combustion is motivated by a large amount of evidence demostrating that mixing rates are controlled by large-scale eddies. Additional motivation is provided by the need to simulate unsteady flows, such as the combustion cycle in a diesel engine; LES is well suited to unsteady combustion problems since it yields time-accurate information.

A common practice in the modeling of nonpremixed combustion is to relate the various chemical mass fractions to a conserved scalar mixture-fraction (Bilger 1980). Chemical reaction rates are known to be strong functions of the mixture fraction, and several models of nonpremixed combustion, such as the Laminar Flamelet Model (LFM) (Peters 1985) and the Conditional Moment Closure (C:MC) (Bilger 1993), take advantage of this to acheive closure of the highly non-linear chemical source terms. Application of such theories in LES involves characterizing the state of mixing within each grid cell. A useful measure of subgrid-scale mixing is the scalar variance, which can either be modeled or clse computed by integrating its transport equation.

An important term in the scalar variance equation is the scalar dissipation rate. This term represents the rate at which reactants are brought together at the molecular level. High rates of dissipation can, in the presence of cold reactants, cause

1 Lawrence Livermore National Laboratory

This work was performed under the auspices of the U.S. Department of

Energy by Lawrence Livermore National Laboratory under contract

No. W-7405-Eng-48. 
flames to extinguish or else fail to ignite. Mixture-fraction-based models of turbulent combustion usually express reaction rates as functions of the scalar dissipation rate. The scalar dissipation rate is a highly intermittent phenomenon, exhibiting large fluctuations associated with the smallest turbulent length scales; however, the net dissipation is determined by the rate at which energy is fed to the turbulence at large (resolvable) scales. Therefore, there is more information available for modeling the scalar dissipation rate in an LES than there is in a Reynolds averaged calculation.

De Bruyn Kops et al. (1998) proposed a model for the subgrid-scale scalar dissipation rate which has the same form as the leading term in a model proposed by Girimaji and Zhou (1996). The model contains a coefficient which can be spatially and temporally dependent. The primary motivation for this work is to determine whether an assumed spectrum methodology can be employed in determining the model coefficient. Another goal is to investigate the accuracy of the model for a flow with large density variations, due to heat release, and a temperature dependent scalar diffusivity.

\section{Definitions}

Consider a turbulent reacting flow in which streams of fuel and oxidizer meet in a combustion chamber where mixing and reaction take place. During the combustion process, many chemical species may be produced and/or destroyed; however, elemental mass fractions are conserved. Let $Z=Z(\mathbf{x}, t)$ be a conserved scalar mixture-fraction, defined as

$$
Z \equiv \frac{\left(Z_{i}-Z_{i, o}\right)}{\left(Z_{i, f}-Z_{i, o}\right)}, i=1,2, \ldots, N
$$

where $Z_{i}$ is the mass fraction of element $i, N$ is the total number of elements and the indicies $f$ and $o$ refer to values in the fuel and oxidizer streams, respectively. It can be seen that $Z=1$ in the fuel stream and $Z=0$ in the oxidant stream. Now let the dissipation rate of scalar fluctuations be denoted by $2 \chi$, where

$$
\chi(\mathbf{x}, t) \equiv D \nabla Z \cdot \nabla^{Z}
$$

Hcre $D$ represents the scalar diffusivity, which may be a function of temperature, i.e., $D=D(T)$. In order to characterize the mean scalar dissipation rate within an LES grid cell, it is necessary to specify the spatial filter associated with the LES mesh. The filter is defined by

$$
\bar{Z}(\mathbf{x}) \equiv \int G\left(\left|\mathbf{x}-\mathbf{x}^{\prime}\right| ; \Delta\right) Z\left(\mathbf{x}^{\prime}\right) d \mathbf{x}^{\prime}
$$

where the integral is taken over all 3-dimensional space (for brevity, the $t$ dependence has been dropped). The filter kernel $G\left(\left|\mathbf{x}-\mathbf{x}^{\prime}\right| ; \Delta\right)$ is normalized,

$$
\int_{-\infty}^{\infty} G(r ; \Delta) d r=1
$$


and has a characteristic width $\Delta$ which is directly related to the grid spacing of the LES mesh. The goal is to derive a model for the filtered value of the densityweighted scalar dissipation rate, i.e., $\overline{\rho \chi}$. Results of de Bruyn Kops et al. (1998) suggest this term be modeled in the following way,

$$
\overline{\rho \chi} \equiv \overline{\rho D \nabla Z \cdot \nabla Z} \approx C \bar{\rho} D(\widetilde{T}) \nabla \widetilde{Z} \cdot \nabla \widetilde{Z}
$$

where a tilde $\widetilde{()}$ is used to denote a Favre-filtered variable, e.g., $\widetilde{Z} \equiv \overline{\rho Z} / \bar{\rho}$. The model contains a coefficient $C$ which can be spatially and temporally dependent, i.e., $C=C(\mathbf{x}, t)$. In the next section, a new method will be described for computing $C(\mathbf{x}, t)$; such that, $(5)$ is correct on average. The method utilizes an assumed form for the $Z$ energy spectrum.

\section{Determination of model coefficient}

In order to relate $C$ to an energy spectrum, $Z$ must be transformable to wavenumber space. Fourier's integral theory assumes that $\int|Z(\mathbf{x})| d \mathbf{x}$ is bounded. For this to be the case, $Z(\mathbf{x})$ will be considered to be zero outside a very large box. The box can be made arbitrarily large so that an assumption of homogeneity may also be made. Forward and inverse Fourier transforms of $Z$ are defined as

$$
\begin{gathered}
\widehat{Z}(\mathbf{k}) \equiv \frac{1}{2 \pi} \int \exp (-i \mathbf{k} \cdot \mathbf{x}) Z(\mathbf{x}) d \mathbf{x}, \\
Z(\mathbf{x}) \equiv \int \exp (i \mathbf{k} \cdot \mathbf{x}) \widehat{Z}(\mathbf{k}) d \mathbf{k},
\end{gathered}
$$

where $\mathbf{k}$ is a wavevector given in radians per unit length. The Fourier transform of $\partial \bar{Z} / \partial x_{j}$ is

$$
\frac{\widehat{\partial \bar{Z}}}{\partial x_{j}}=-i k_{j} \widehat{G}(k ; \Delta) \widehat{Z}(\mathbf{k})
$$

where $\widehat{G}$ is a function only of the magnitude of $\mathbf{k}$, i.e., $k^{2} \equiv \mathbf{k} \cdot \mathbf{k}=k_{j} k_{j}$. Writing $\partial \bar{Z} / \partial x_{j}$ as the inverse transform of (8) and squaring both sides (and summing on j) leads to

$$
\left|\frac{\partial \bar{Z}}{\partial x_{j}}\right|^{2}=\iint \exp \left(i\left(\mathbf{k}+\mathbf{k}^{\prime}\right) \cdot \mathbf{x}\right)\left(-k_{j} k_{j}^{\prime}\right) \widehat{G}(k ; \Delta) \widehat{G}\left(k^{\prime} ; \Delta\right) \widehat{Z}(\mathbf{k}) \widehat{Z}\left(\mathbf{k}^{\prime}\right) d \mathbf{k} d \mathbf{k}^{\prime} .
$$

Since $Z(\mathbf{x})$ is real, $\widehat{Z}\left(\mathbf{k}^{\prime}\right)=\widehat{Z}^{*}\left(-\mathbf{k}^{\prime}\right)$ where the asterisk denotes the complex conjugate. For homogeneous turbulence, the Fourier amplitudes $\widehat{Z}(\mathbf{k})$ and $\widehat{Z}^{*}\left(-\mathbf{k}^{\prime}\right)$ are statistically orthogonal; hence, the enscmblc average $\left\langle\widehat{Z}(\mathbf{k}) \widehat{Z}^{*}\left(-\mathbf{k}^{\prime}\right)\right\rangle$ is zero unless $-\mathbf{k}^{\prime}=\mathbf{k}$ (Batchelor 1953). The ensemble average of (9) thus becomes

$$
\left\langle\frac{\partial \bar{Z}}{\partial x_{j}} \frac{\partial \bar{Z}}{\partial x_{j}}\right\rangle=\int k^{2} \widehat{G}^{2}(k ; \Delta)\left\langle\widehat{Z}^{*}(\mathbf{k}) \widehat{Z}(\mathbf{k})\right\rangle d \mathbf{k} .
$$


The integral in (10) may be cast as an integral over a spherical shell of radius $k$, followed by integration over all shells. For example, if $d \sigma$ denotes a differential surface element of a shell, then (10) can be written

$$
\int k^{2} \widehat{G}^{2}(k ; \Delta)\left\langle\widehat{Z}^{*}(\mathbf{k}) \widehat{Z}(\mathbf{k})\right\rangle d \mathbf{k}=\int_{0}^{\infty} k^{2} \widehat{G}^{2}(k ; \Delta) \oint\left\langle\widehat{Z}^{*}(\mathbf{k}) \widehat{Z}(\mathbf{k})\right\rangle d \sigma d k .
$$

The shell integral in (11) is equal to twice the three-dimensional, scalar energy spectrum, i.e., $2 E_{Z}(k)$; hence,

$$
\left\langle\frac{\partial \bar{Z}}{\partial x_{j}} \frac{\partial \bar{Z}}{\partial x_{j}}\right\rangle=2 \int_{0}^{\infty} k^{2} \widehat{G}^{2}(k ; \Delta) E_{Z}(k) d k .
$$

Repcating the analysis for $\partial Z / \partial x_{j}$ leads to the result

$$
\left\langle\overline{\frac{\partial Z}{\partial x_{j}} \frac{\partial Z}{\partial x_{j}}}\right\rangle=\left\langle\frac{\partial Z}{\partial x_{j}} \frac{\partial Z}{\partial x_{j}}\right\rangle=2 \int_{0}^{\infty} k^{2} E_{Z}(k) d k .
$$

The coefficient $C$ can be determined by taking the average of (5), i.e.,

$$
C=\frac{\langle\overline{\rho D \nabla Z \cdot \nabla Z}\rangle}{\langle\bar{\rho} D(\widetilde{T}) \nabla \widetilde{Z} \cdot \nabla \widetilde{Z}\rangle} \approx \frac{\langle\overline{\nabla Z \cdot \nabla Z}\rangle}{\langle\nabla \bar{Z} \cdot \nabla \bar{Z}\rangle}=\frac{\int_{0}^{\infty} k^{2} E_{Z}(k) d k}{\int_{0}^{\infty} k^{2} \widehat{G}^{2}(k ; \Delta) E_{Z}(k) d k} .
$$

Here it has been assumed that $\rho D$ approximately cancels in the numerator and denominator and that $\langle\nabla \widetilde{Z} \cdot \nabla \widetilde{Z}\rangle \approx\langle\nabla \bar{Z} \cdot \nabla \bar{Z}\rangle$.

\section{Assumed energy spectrum}

In order to compute $C$ using (14), it is necessary to specify the functions $\widehat{G}(k ; \Delta)$ and $E_{Z}(k)$. For the present analysis, the LES grid filter is assumed to be a 'top-hat' function, which, in Fourier space, is defined as

$$
\widehat{G}=\frac{2 \sin (k \Delta / 2)}{k \Delta} .
$$

The scalar energy spectrum is assumed to be that of isotropic turbulence at high Reynolds number (Tennekes and Lumley 1972), i.e.,

$$
E_{Z}(k)=A \exp \left[-1.73\left(k l_{Z}\right)^{-4 / 3}\right] k^{-5 / 3} \exp \left[-2.25\left(k \eta_{Z}\right)^{4 / 3}\right],
$$

which is applicable for fluids with Schmidt numbers near unity. For fluids with a very low or very high Schmidt number, an inertial-diffusive or viscous-convective subrange should be included in the assumed spectrum. Equation (16) contains three unknown parameters: a constant $A$, a scalar integral scale $l_{Z}$, and a dissipation scale $\eta_{Z}$ (Corrsin 1951). The constant $A$ divides out of (14), and hence, is irrelevant to the modeling. The length scales $l_{Z}$ and $\eta_{Z}$ determine roughly where 
the spectrum peaks and where it goes to zero. If $l_{Z}$ and $\eta_{Z}$ are estimated locally and/or instantaneously, then $C$ will be obtained as a function of space and/or time. In the present analysis, $l_{Z}$ and $\eta_{Z}$ are assumed to be related to the integral scale $l$ and Kolmogorov microscale $\eta$ of the velocity field $u_{i}$. This implies that the flow is sufficiently developed; such that, the statistics of the velocity field are reflected in the scalar field. The length scale estimates are as follows:

$$
\begin{gathered}
l_{Z} \approx l \approx \frac{V}{\left\langle S_{i j} S_{i j} / 9\right\rangle^{1 / 2}}, \\
V \equiv\left\langle\widetilde{u}_{i} \widetilde{u}_{i}\right\rangle^{1 / 2} \\
S_{i j} \equiv \frac{1}{2}\left(\frac{\partial \widetilde{u}_{i}}{\partial x_{j}}+\frac{\partial \widetilde{u}_{j}}{\partial x_{i}}\right), \\
\eta_{Z} \approx \eta S c^{-1 / 2}, \\
\eta \approx \alpha l R e_{l}^{-3 / 4}, \\
R e_{l} \equiv l V /\langle\nu\rangle, \\
\langle\nu\rangle \equiv S c\langle D(\widetilde{T})\rangle .
\end{gathered}
$$

where, $S c$ is the global Schmidt number and $\alpha$ is a constant.

In the present analysis, $\alpha$ was set to 2 in order to bring the model into agreement with the DNS data (to be described in the next section). The estimates for $\eta Z$ and $\eta$ are strictly valid only at high Reynolds number. Due to the low Reynolds number of DNS, the dissipation range makes up most of the energy spectrum; thus, in a prior $i$ tests, $C$ can be sensitive to the estimate for $\eta_{Z}$. In a high Reynolds number flow, however, the energy in the dissipation range contributes only slightly to the integral of the spectrum. Hence, there is good reason to expect that, at higher Reynolds numbers, $C(x, t)$ will be less sensitive to the estimate for $\eta_{Z}$, so that $\alpha$ can probably be set to 1 if the Reynolds number is high enough.

\section{DNS data}

The model was tested using data from DNS of a reacting temporal mixing layer (Bushe et al. 1998). The DNS utilized a computational mesh consisting of $240 \times$ $120 \times 120$ points in the $x, y$ and $z$ directions, respectively. It was initialized with a planar laminar flame centered in the domain. Isotropic turbulent velocity fluctuations, taken from a previous simulation of forced incompressible turbulence, were superimposed on the flow induced by the heat release of the flame. Periodic boundaries were imposed in the $y$ and $z$ directions. Outflow boundary conditions, as described in Poinsot and Lele (1992), were employed in the $x$ direction to allow for expansion due to heat release. In order to avoid unphysical generation of vorticity by the imposed outflow conditions, the velocity fluctuations were filtered to zero at the $x$ boundaries. A two-step reduced chemical kinetic mechanism for methane 

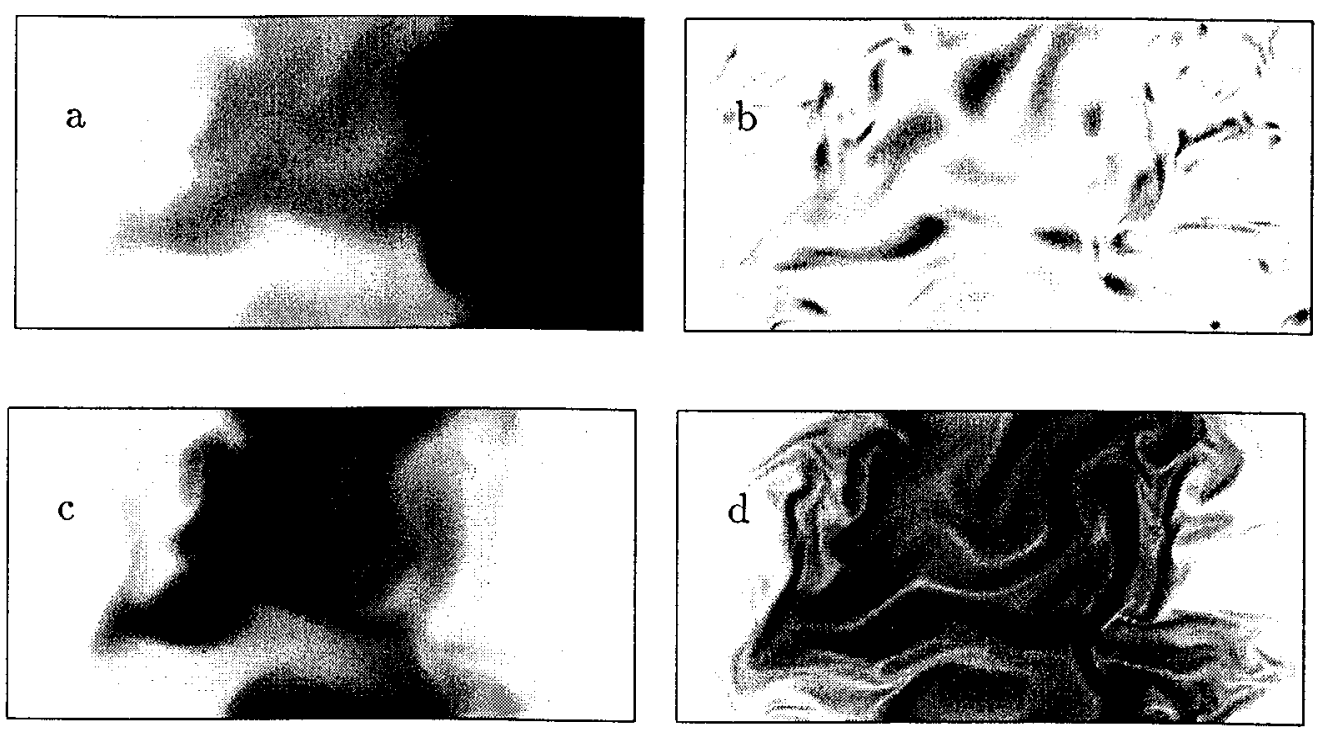

FIGURE 1. Grayscale plots of (a) Mixture-fraction, (b) Enstrophy, (c) Temperature and (d) Logarithm of scalar dissipation rate on an $x-y$ plane in the DNS flow field at $t=15$.

was used with a further step added to approximate $\mathrm{NO}_{\mathrm{x}}$ chemistry. The maximum possible density ratio, based on the adiabatic flame temperature, was 7.4 ; the maximum density ratio acheived in the simulation was 6.3. The global acoustic Reynolds number was $R e_{a}=2000$, and, for the case used here, the Schmidt number of all species was set to $S c=0.75$. The ratio of specific heats was set to $\gamma=1.3$, and the scalar diffusivity was prescribed as the following function of temperature

$$
D=\frac{[(\gamma-1) T]^{0.76}}{\operatorname{Re} S c} .
$$

Figure la shows the mixture-fraction field on a slice in the three-dimensional domain after the flow had evolved for 15.0 acoustic time units. The gas on the right is fuel and the gas on the left is oxidant. The enstrophy field on the same slice is shown in figure 1b. Figure 1c shows the tempcrature field resulting from the exothermicity of the reactions. The scalar dissipation rate is shown in figure 1d; it is clear that this field has structures associated with fine length scales.

\section{Results}

In the a priori tests, the DNS data were averaged onto a $24 \times 12 \times 12$ point LES grid such that each LES grid cell was comprised of $10^{3}$ DNS data points. Ensemble averages were approximated by averaging in the homogeneous directions, i.e., over $(y-z)$ planes. The model coefficient $C$ was thus computed as a function of the inhomogeneous direction $x$ and time $t$.

Figure 2 shows the true and modelcd values of $C$ as a function of $x$ at four different times in the simulation. At $t=7.5, C$ is overpredicted. This is due to the fact that insufficient time has elapsed for a turbulent spectrum to have developed for the $Z$ 

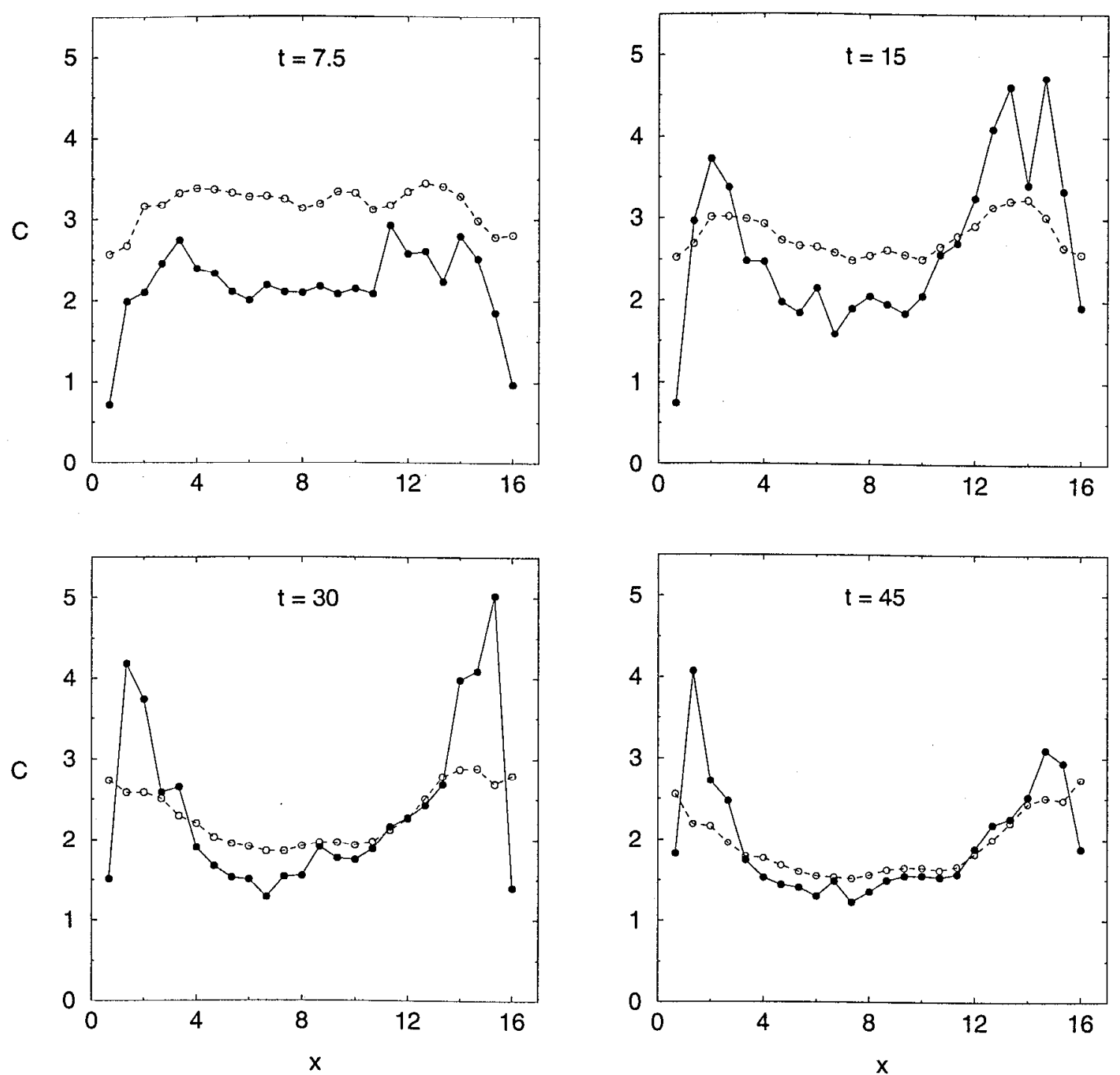

FIGURE 2. Coefficient in model for $\overline{\rho \chi}$ as a function of $x$ and $t$ from DNS of temporal reacting mixing layer: • - DNS; 0 - assumed spectrum method.

field. However, as time elapses, the model for $C(x, t)$ becomes increasingly more accurate. Furthermore, it is encouraging that the model appears most accurate near the middle of the domain where the bulk of the reactions are occuring. The large oscillations in the DNS values of $C(x, t)$ near the ends of the domain are due to the fact that $Z(\mathbf{x}, t)$ is nearly constant close to the boundaries, such that the scalar dissipation rates are very small. Computation of the DNS values for $C$ near the boundaries involves taking the ratio of two very small numbers; hence, the results are subject to numerical noise.

In figure 3 , true versus estimated values of $\overline{\rho \chi}$ are plotted for every point on the LES grid. The model values were computed from (5) using $C$ as shown in figure 1, i.e., $\overline{\rho \chi}_{\text {est }}=C(x, t) \bar{\rho} D(\widetilde{T}) \nabla \widetilde{Z} \cdot \nabla \widetilde{Z}$. The results show good agreement between the 

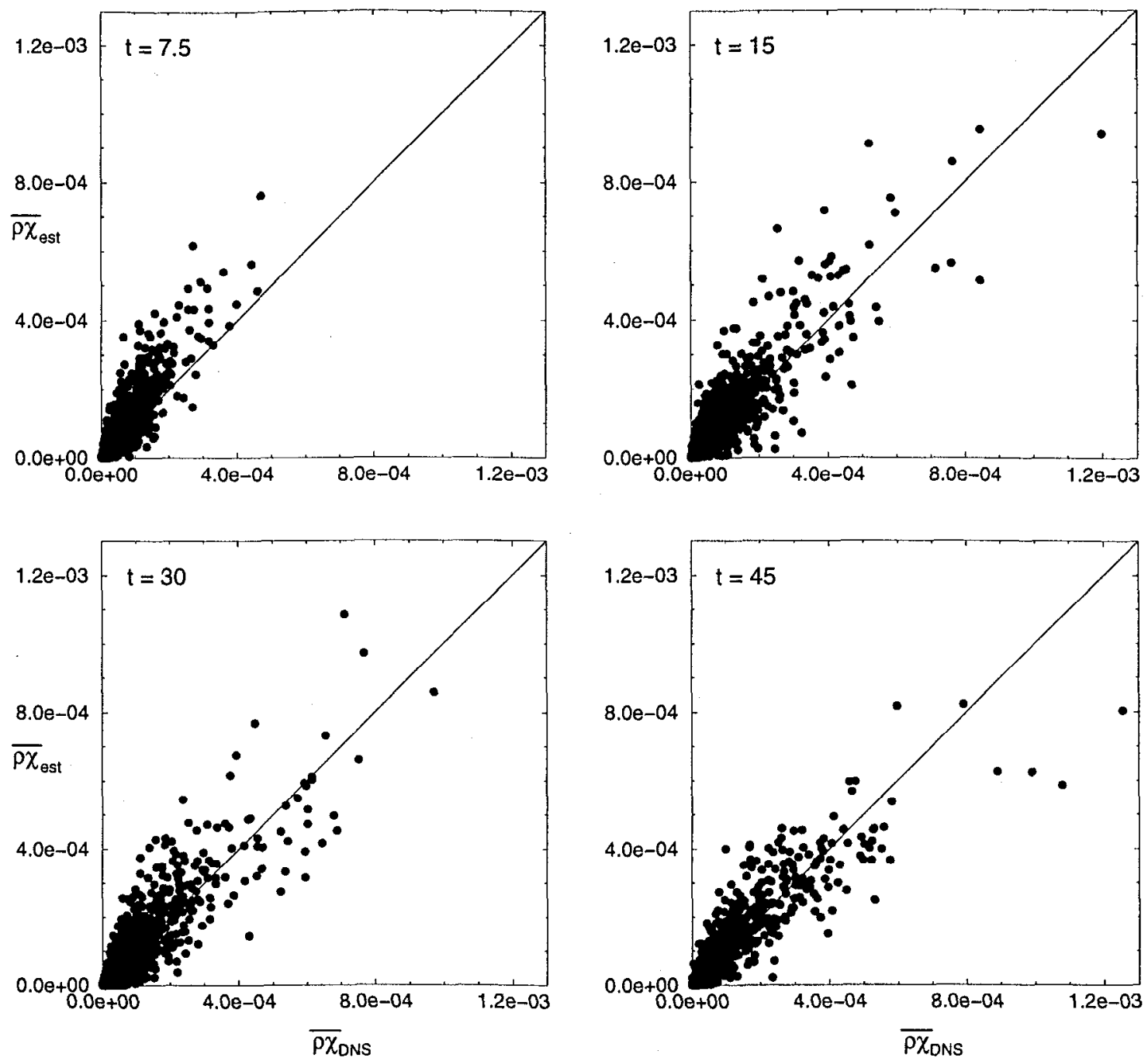

FIguRE 3. Estimated (est) versus true (DNS) values of $\overline{\rho \chi}$, computed by filtering $240 \times 120 \times 120$ point DNS data onto $24 \times 12 \times 12$ point LES grid.

true and estimated values of $\overline{\rho \chi}$. Linear correlation coefficients for $\overline{\rho \chi}_{\text {est }}$ and $\bar{\rho}_{\text {DNS }}$ are: $0.911,0.897,0.898$ and 0.913 for $t=7.5,15,30$ and 40 , respectively.

\section{Conclusions}

A model has been presented for the scalar dissipation rate in nonpremixed turbulent combustion. The model contains a coefficient which can be computed by assuming a form for the scalar energy spectrum, The scalar integral scale $l_{Z}$ and dissipation length scale $\eta_{Z}$ appear as parameters in the assumed spectrum. The modcl was cvaluatcd a priori using DNS results for a turbulent reacting mixing layer. It was found that the assumed spectrum, when combined with local estimates for $l_{Z}$ and $\eta_{Z}$, gave accurate predictions of the model coefficient, including its spatial and temporal variations. Furthermore, the tests revealed a high correlation between true and modeled values of the scalar dissipation rate, which appears 
to validate the model for turbulent reacting flows in the presence of heat release.

\section{Acknowledgements}

Partial support for this work was provided by the U.S. Department of Energy through Lawrence Livermore National Laboratory under Contract No. W-7405Eng-48.

\section{REFERENCES}

Batchelor, G. K. 1953 The Theory of Homogeneous Turbulence. Cambridge University Press.

Bilger, R. W. 1980 Turbulent Flows with Nonpremixed Reactants. in Topics in Applied Physics vol. 44, chap. 3, pp. 2-36. Berlin: Springer-Verlag.

Bilger, R. W. 1993a Conditional moment closure for turbulent reacting flow. Phys. Fluids A. 5(2), 436.

Bushe, W. K., Bilger, R. W. \& Ruetsch, G. R. 1998 Direct Numerical Simulation of nonpremixed combustion with realistic chemistry. To be submitted to J. Fluid Mech.

CoRrsin, S. 1951 On the spectrum of isotropic temperature fluctuations in isotropic turbulence. J. App. Phys.. 25, 657.

de Bruyn Kops, S. M., Riley, J. J., Kosaly, G. \& Cook, A. W. 1998 Investigation of modeling for non-premixed turbulent combustion. Submitted to Flow, Turbulence and Combustion.

GirimaJi, S. S. \& ZHOU, Y. 1996 Analysis and modeling of subgrid scalar mixing using numerical data. Phys. Fluids. 8, 1224-1236.

Peters, N. 1984 Laminar diffusion flamelet models. Prog. Energy Combust. Sci. $10,319$.

Poinsot, T., \& Lele, S. 1992 Boundary conditions for direct simulations of compressible viscous flows. J. Comp. Phys. 101, 104.

Tennekes, H. \& Lumley, J. L. 1972 A First Course in Turbulence. Cambridge: MIT Press. 